\title{
Les migrations à la frontière jurassienne franco- suisse : bassins de vie et bassins d'emploi transfrontaliers
}

Migrations around the Franco-Swiss Jura border : cross-border residential and employment areas

Wanderungen an der französisch-schweizerische Jurabogens Grenze : grenzüberschreitende Wohn - und Arbeitsbereiche

\section{Alexandre Moine}

\section{OpenEdition}

Journals

Édition électronique

URL : http://journals.openedition.org/rge/4312

DOI : $10.4000 /$ rge. 4312

ISSN : 2108-6478

Éditeur

Association des géographes de l'Est

Édition imprimée

Date de publication : 1 septembre 1999

ISSN : 0035-3213

\section{Référence électronique}

Alexandre Moine, «Les migrations à la frontière jurassienne franco-suisse : bassins de vie et bassins d'emploi transfrontaliers », Revue Géographique de l'Est [En ligne], vol. 39 / 4 | 1999, mis en ligne le 13 août 2013, consulté le 10 décembre 2020. URL : http://journals.openedition.org/rge/4312 ; DOI :

https://doi.org/10.4000/rge.4312

Ce document a été généré automatiquement le 10 décembre 2020.

Tous droits réservés 


\title{
Les migrations à la frontière jurassienne franco-suisse : bassins de vie et bassins d'emploi transfrontaliers
}

\author{
Migrations around the Franco-Swiss Jura border : cross-border residential and \\ employment areas \\ Wanderungen an der französisch-schweizerische Jurabogens Grenze : \\ grenzüberschreitende Wohn - und Arbeitsbereiche
}

Alexandre Moine

1 De plus en plus, les frontières catalysent les déplacements, générant et concentrant les mouvements de biens et surtout de personnes. Les déplacements saisis à une échelle locale sont facilement assimilables à des migrations temporaires. Ils sont complexes, reposent sur les décisions de multiples acteurs et se traduisent spatialement par une organisation spécifique de certains espaces transfrontaliers. Dans l'arc jurassien franco-suisse, l'ensemble des mouvements focalisés autour des points de franchissements frontaliers se fonde sur deux réalités fortes, l'une structurelle, la similitude des spécialisations industrielles de part et d'autre de la frontière, l'autre conjoncturelle, le différentiel monétaire important entre les deux pays.

2 Connaître l'origine et la destination de ces déplacements, comprendre leurs fondements doit permettre de mieux les gérer. En se multipliant, les migrations transfrontalières locales posent le problème de la congestion des réseaux de transport, particulièrement dans l'Arc jurassien, de l'augmentation de la pollution et des risques d'accident, mais traduisent également la vitalité des échanges qui constituent de véritables bouffées d'oxygène pour les pays concernés.

Grâce à des exemples précis relevés tout au long de la frontière, nous désirons montrer comment se structurent les relations, quelles sont leurs origines, ce qu'elles impliquent 
en termes d'organisation spatiale et de quelle manière se répartissent les mouvements de personnes.

\section{Le mouvement des navetteurs frontaliers fait clairement apparaître des zones d'emploi transfrontalières}

4 Le développement du travail frontalier est relativement récent si on le considère dans son ampleur actuelle. La forte croissance remonte aux années 1985/1990 au cours desquelles le nombre de frontaliers franc-comtois travaillant dans les quatre cantons suisses frontaliers a été multiplié par près de 2,5 . Au plus fort de la vague d'embauche, en 1991, le nombre de permis frontaliers accordés aux franc-comtois avoisinait les 16 500 unités $[1,2,3,4]$ et se répartissaient principalement entre :

- Belfort et le District urbain du Pays de Montbéliard (plus de 3000 frontaliers), principalement à destination du district de Porrentruy ;

- le canton de Morteau (environ 2900 frontaliers), à destination du canton de Neuchâtel et tout particulièrement des villes du Locle et de la Chaux-de-Fonds ;

- le canton de Pontarlier (environ 2600 frontaliers), à destination des cantons de Neuchâtel

(Val de Travers) et de Vaud (Yverdon, Orbe, La Vallée) ;

- le canton de Morez (1 700 frontaliers), essentiellement à destination de La Vallée.

On dénombre donc cinq points de passage principaux par l'intensité et la diversité de leur trafic (frontaliers, consommateurs, transit international), du sud au nord (fig. 1) : Les Rousses vers La Cure (Canton de Morez vers district de Nyon), Jougne vers Vallorbe (canton de Pontarlier vers district de Vallorbe), Villers-le-Lac vers Le Locle (canton de Morteau vers district du Locle), Nord Franche-Comté vers Porrentruy (cantons de Delle et Hérimoncourt vers district de Porrentruy), et huit points de passage secondaires: Bois-d'Amont vers Le Brassus, Mouthe vers La Vallée, Les Fourgs vers l'Auberson, Biaufond vers La Chaux-de-Fonds, Goumois vers Saignelégier, Villard-lès-Blamont et Brémoncourt, respectivement vers Damvant et La Motte. 
Fig. 1 : Les principaux passages frontaliers suivant l'importance des flux moyens, journaliers, de véhicules en 1997

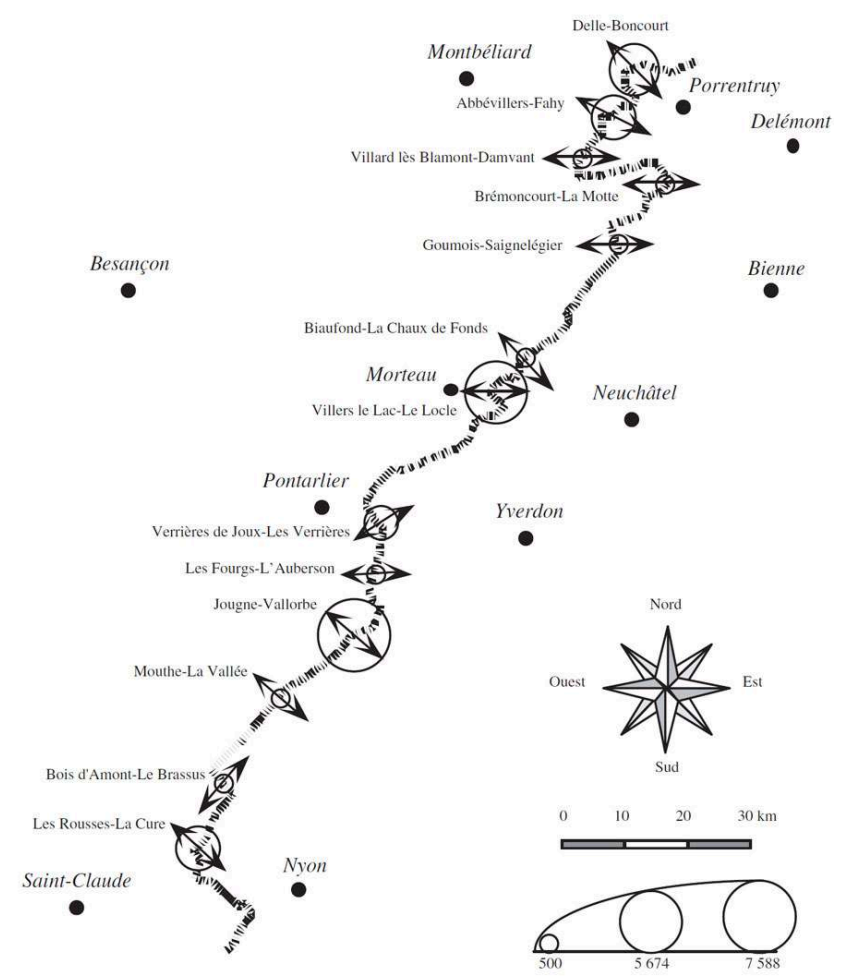

Source : Direction Régionale de l'Équipement

6 On relève par conséquent des auréoles très nettes autour des points de franchissement frontaliers, concentrant cette main-d'œuvre encline à minimiser la distance domiciletravail et théoriquement obligée de résider à moins de $25 \mathrm{~km}$ de la frontière. En regard, de l'autre côté de la frontière, on observe une relative concentration des entreprises autour des mêmes points de franchissement, l'objectif étant de profiter de la maind'œuvre française qualifiée et moins exigeante d'un point de vue salarial (fig. 2). 
Fig. 2 : Une structuration de l'espace frontalier autour des points de franchissement : l'exemple des pôles de Pontarlier-Vallorbe et Morteau-Le Locle-La Chaux de Fonds.

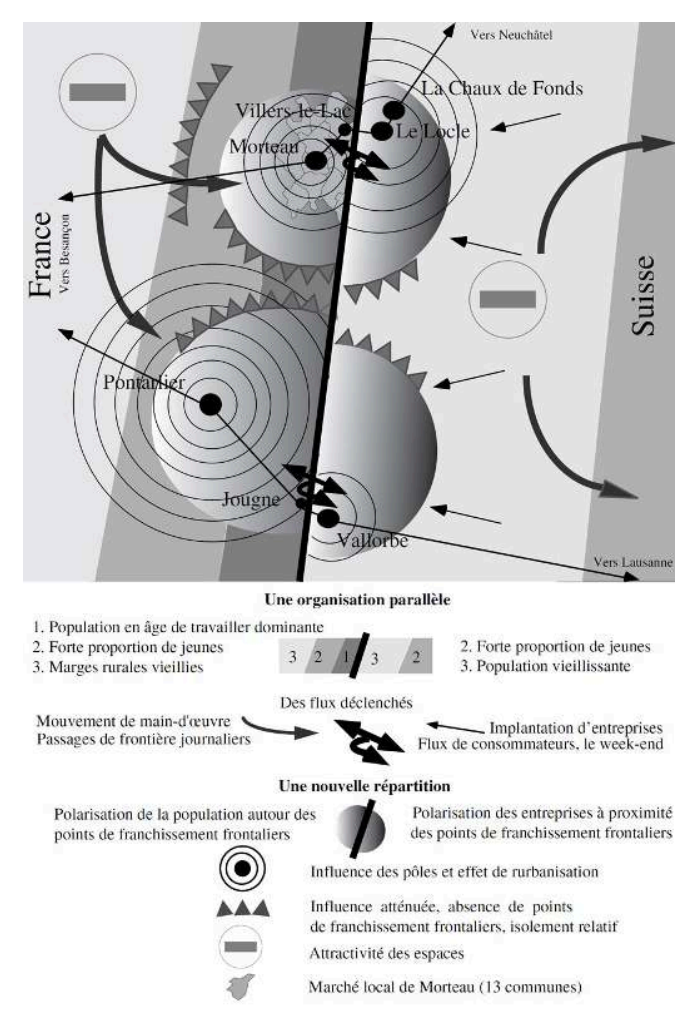

7 Les mouvements des navetteurs frontaliers révèlent l'existence de bassins d'emplois transfrontaliers, qui demeurent cependant théoriques, puisque, dans les faits nous n'observons au mieux que la juxtaposition de bassins d'emplois français et suisses dont le fonctionnement n'est que partiellement intégré (couverture sociale, impôts, chômage).

\section{Le travail à façon détermine de nombreux échanges de pièces destinées à la construction micromécanique}

Les relations industrielles transfrontalières sont localement relativement importantes. Deux indicateurs peuvent nous aider à le montrer : les flux téléphoniques tout d'abord, puisqu'en 1989, 30 à $50 \%$ des appels vers l'étranger à partir des zones tarifaires de Morez et de Pontarlier concernaient la Suisse, cette part atteignant $75 \%$ pour la zone de Morteau, et seulement 15 à $20 \%$ pour celles de Belfort et de Montbéliard. Il est à noter toutefois que ce phénomène, bien qu'important, est sans commune mesure avec ce que l'on peut observer autour de l'agglomération genevoise, puisque dans l'arc jurassien il ne concerne qu'une étroite bande frontalière, autour des principaux points de passage.

9 L'analyse du volume de produits échangés entre la Franche-Comté et la Suisse conforte l'hypothèse que la frontière joue un rôle important en rapprochant les entreprises : depuis dix ans, les échanges entre la Franche-Comté et la Suisse se sont accentués (tableau 1) en renforçant la complémentarité économique. Entre 1985 et 1994, les exportations de la Franche-Comté vers la Suisse sont passées de 1,4 à 2,6 milliards de francs français [5]. Ainsi, le Doubs réalise plus de $10 \%$ de ses échanges avec la Suisse [6], 
avec une forte spécialisation dans l'activité des instruments et matériels de précision (53,5\% des importations en provenance de Suisse et $41 \%$ des exportations vers la Suisse en 1993), révélant une forte complémentarité autour d'une même filière de production comme dans le cas de l'horlogerie. Ces échanges s'effectuent en grande partie entre des PME, localisées dans les cantons frontaliers (Morteau, Maîche, Le Russey). Pour le département du Jura, les échanges sont légèrement plus faibles et portent sur d'autres activités : les jeux et jouets (importation de Suisse), les produits chimiques de base et les jeux et jouets (exportation vers la Suisse).

Tableau 1 : Évolution de la part de la Suisse dans les échanges extérieurs des départements frontaliers (en \%)

\begin{tabular}{|l|c|c|c|c|c|}
\hline & & Doubs & Jura & H-Saône & T. de B. \\
\hline \multirow{2}{*}{ Importations } & 1984 & 14,86 & 7,83 & 3,05 & 5,66 \\
& 1993 & 16,04 & 5,86 & 1,88 & 5,23 \\
Exportations & 1984 & 5,91 & 12,07 & 1,89 & 3,01 \\
& 1993 & 10.68 & 13,36 & 3,22 & 2,00 \\
\hline \multicolumn{2}{l}{ Source : Daviot (J.-M.), Gillon (P.), 1995. } \\
\hline
\end{tabular}

Le partenariat avec des entreprises suisses concerne principalement les cantons où le secteur des microtechniques et plus particulièrement de l'horlogerie sont implantés, révélant les liens étroits qui se sont noués autour du travail à façon ${ }^{1}$ (les pièces sont conçues en Suisse, usinées en France et définitivement assemblées en Suisse pour produire des mécanismes estampillés "Swiss made»). L'origine de ces relations dans l'horlogerie, en partie fondées sur des rapports de domination/dépendance, tient à une adaptation très différente des entreprises françaises et suisses à la montée de la concurrence asiatique dans le milieu des années 70 . Les premières se sont malheureusement orientées vers une vaine concurrence, alors que les entreprises suisses misaient sur le maintien d'une production de haut de gamme [7] qui leur a permis de surmonter la crise horlogère. Ainsi, les relations entre les entreprises françaises et suisses, principalement dans le domaine de l'horlogerie, sont aujourd'hui nettement dominées par des rapports de sous-traitance, au détriment de la France (la moitié des entreprises sont concernées contre seulement un quart en Suisse), alors que la Suisse se consacre plus à l'exportation du produit fini. Ces relations expliquent de nombreux mouvements de transporteurs locaux entre France et Suisse qui, d'ailleurs, ne déclarent pas toujours la réalité des marchandises transportées afin d'éviter les démarches administratives et pour contourner certaines incohérences, tels les horaires d'ouverture non synchronisés des postes de dédouanement.

\section{Le tourisme d'achat profite des avantages de l'Europe hors d'une Suisse hyper-protégée}

11 Le tourisme d'achat est de plus en plus prisé par les citoyens helvétiques qui trouvent en France des prix dans l'alimentation plus bas qu'en Suisse, puisqu'ils sont indexés sur les prix européens. En 1996, les Suisses ont acheté pour 1,5 milliard de francs suisses hors des frontières ${ }^{2}$ et le phénomène ne fait que s'amplifier compte tenu de la récession qui affecte aujourd'hui la Suisse. La conséquence est double, le commerce intérieur de 
la Suisse se contracte, tandis que dans les régions frontalières limitrophes, les grandessurfaces se multiplient et certains types de services sont particulièrement sollicités.

Une enquête menée pour le compte de la Télévision Suisse Romande ${ }^{3}$ portant sur l'équivalent d'un caddie d'une soixantaine d'articles de consommation courante, a révélé des différences de prix considérables. À la Coop des Portes Rouges à Neuchâtel les 60 articles ont été payés 910,40 F, alors qu'au Géant Casino de Pontarlier distant de $55 \mathrm{~km}$, ils n'ont coûté que 593,76 F soit 34,8\% moins cher. Le Magro de Marins (NE) vend ce même caddie 923,12 $\mathrm{F}$ contre 606,11 $\mathrm{F}$ à l'Intermarché de Morteau situé à 45 $\mathrm{km}$, la différence étant encore ici de $34,3 \%$.

Ce tourisme se traduit par de nombreuses migrations locales des frontaliers suisses vers les principales villes du Haut-Doubs, Maîche, Morteau ou Pontarlier. Nous avons donc mené une enquête sur le terrain ${ }^{4}$ afin de connaître l'importance des consommateurs suisses dans deux hypermarchés frontaliers français à Pontarlier et à Morteau; les résultats sont saisissants. L'enquête s'est déroulée en deux phases, une première série à l'approche des fêtes de fin d'année les 13, 19 et 20 décembre 1997, une deuxième série les 24 et 25 avril 1998 en période de consommation courante. On constate que la part des véhicules immatriculés en Suisse varie entre 20 et près de $50 \%$, avec un très net renforcement du phénomène dans les périodes qui précédent les fêtes.

14 - Pour ce qui concerne l'Intermarché de Morteau 162 véhicules suisses ont été recensés le 13 décembre 1997 et 258 le 25 avril 1998. L'aire de chalandise s'étend principalement sur la bande frontalière puisqu'on recense de nombreux véhicules en provenance du canton de Neuchâtel (78\%), particulièrement du Locle et de La Chaux-de-Fonds, mais également de plus loin, en provenance des cantons du Jura (10\%) et de Berne (8\%).

15 - Au Géant-Casino de Pontarlier, 839 véhicules suisses ont été recensés les 19 et 20 décembre 1997 et 131 véhicules le 24 avril 1998. L'origine des consommateurs se partage principalement entre deux cantons, celui de Neuchâtel (56\%) - cela concerne particulièrement le Val de Travers - et celui de Vaud (32\%) dans une moindre mesure ceux de Fribourg (5\%), Berne (4 \%) et Genève (1,5\%).

16 Les aires de chalandises ainsi esquissées sont dissymétriques ${ }^{5}$ (fig. 2), au profit de l'espace frontalier français. La raison est simple, côté français les pôles frontaliers rayonnent sur des aires plus importantes dans la mesure où ils sont éloignés des villes importantes situées dans la zone de piémont et situés en zone rurale. Côté suisse, par contre, l'aire de chalandise est plus réduite car si la concurrence ne s'inscrivait pas en termes de diversité de services ou de produits, les pôles frontaliers français n'auraient aucune chance face à l'offre en Suisse ; l'aire de chalandise se manifeste par les prix avantageux que l'éloignement à la frontière péjore rapidement.

La fréquence de ces achats est, dans la plupart des cas, hebdomadaire, les déplacements ayant lieu de préférence le vendredi et surtout le samedi, ainsi que les jours fériés. Pour les commerces frontaliers français, ces migrations sont providentielles dans une région à la fois rurale et industrielle où les salaires sont inférieurs à la moyenne nationale, seulement soutenus par la présence de frontaliers. Loin d'être une zone franche, la zone frontalière du Haut-Doubs est en fait constituée d'une succession de bassins de vie transfrontaliers que ces migrations ne cessent de révéler (fig. 3). 
Fig. 3 : Les bassins de vie transfrontaliers

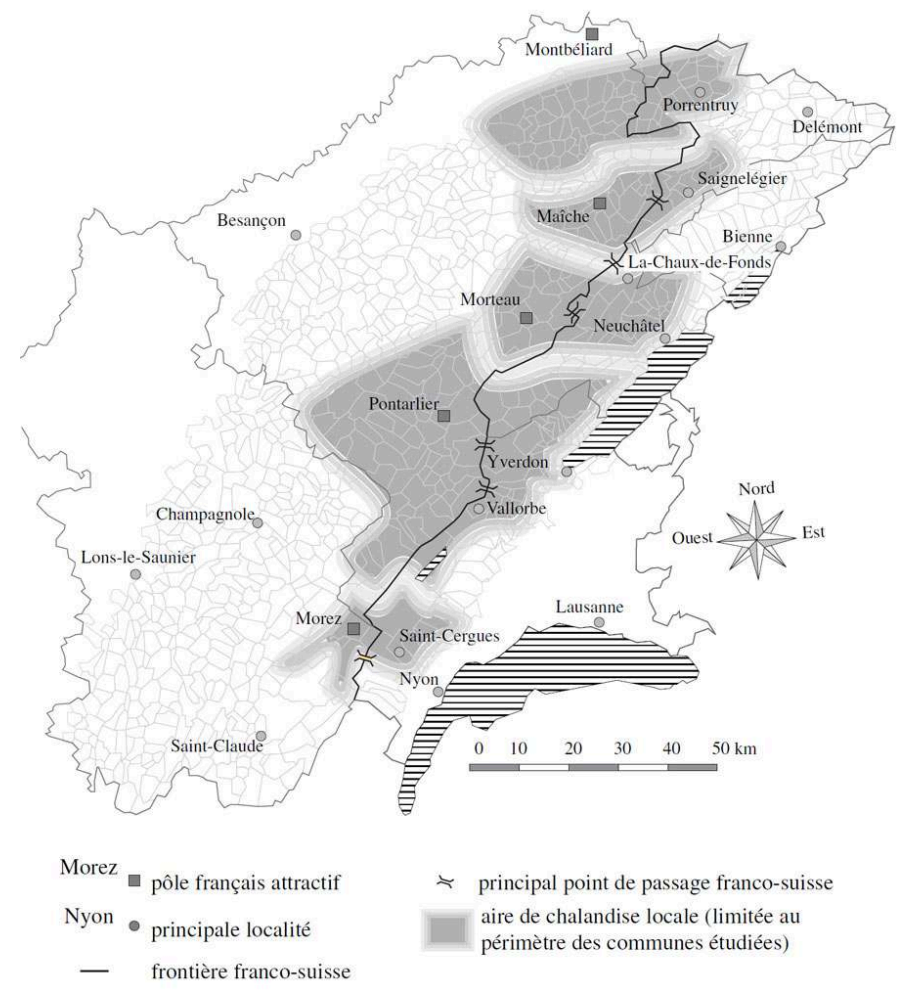

\section{L'accès aux services médicaux en France pour contourner un système de protection suisse hors de prix}

Les commerces ne sont pas les seuls services utilisés par les consommateurs suisses, les services médicaux sont également fortement sollicités, et ce pour deux raisons principales : d'une part, la Suisse ne fait plus aujourd'hui figure d'exception parmi les pays touchés par la crise, l'augmentation du chômage y est très sensible, le taux ayant évolué de moins de $1 \%$ à plus de $5 \%$ depuis trois ans, avec une paupérisation sensible des populations concernées compte tenu de la faiblesse de l'assurance chômage; d'autre part la couverture maladie se dégrade rapidement en Suisse. Suite aux nouvelles dispositions de la Loi Maladie (1996), les cotisations aux mutuelles ont augmenté de $20 \%$ en 1996-1997 et risquent de subir une hausse identique dans les années à venir ; en outre, certains frais médicaux ne sont pas remboursés.

L'effet est indéniable sur l'augmentation du nombre de médecins et de dentistes installés à proximité des principaux points de passage frontaliers en France. Une enquête ${ }^{6}$ menée par nos soins en 1997 révèle que par rapport à l'Inventaire Communal de 1988 l'offre de services médicaux a fortement évolué en zone frontalière indépendamment de l'évolution structurelle régionale (fig. 4). 
Fig. 4 : La Suisse malade de son système de protection sociale pour le bénéfice des praticiens français

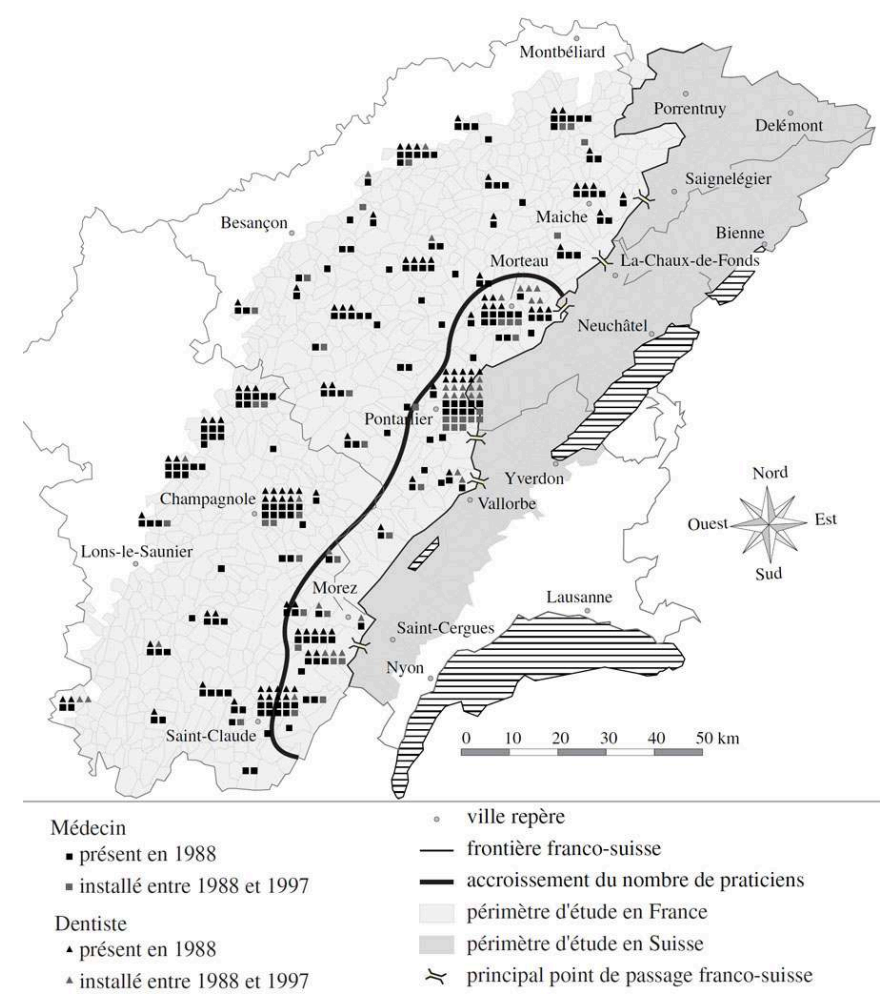

Nous avons ainsi relevé 48 dentistes en 1988 sur la zone frontalière allant du canton de Saint-Hippolyte à celui de Saint-Claude, soit un dentiste pour 2500 habitants, tandis que le reste du massif jurassien (24 cantons) en comptait 66, soit une proportion rapportée à la population strictement identique. Après avoir procédé à une enquête par minitel, nous constatons qu'en 1997, le nombre de dentistes pour la même zone frontalière est passé à 72 , soit une hausse de $50 \%$, alors que dans le reste du massif le nombre est resté le même (fig. 4)!

21 C'est Pontarlier qui bénéficie le plus de cette hausse puisque l'offre passe de 9 à plus de 20 dentistes, sans compter les installations à Jougne ou aux Hôpitaux-Neufs. La situation est, dans une moindre mesure, la même à Morteau et Villers-le-Lac, ainsi qu'aux Rousses. Les installations de cabinets dans les villages ruraux situés à proximité des points de franchissement (Jougne, Les Hôpitaux-Neufs) traduisent clairement l'intérêt que suscite la présence des consommateurs suisses auprès des praticiens français.

Suivant la même logique, nous avons recensé les médecins généralistes, et constaté une hausse importante de leur nombre en zone frontalière $(+30 \%)$, qui profite toujours à Pontarlier, alors que leur nombre n'évolue que de $10 \%$ sur le reste du massif.

L'attractivité des services médicaux français est par conséquent forte, au gré des politiques menées de part et d'autre de la frontière, et s'étend sur une aire de chalandise qui atteint le littoral neuchâtelois, la vallée de Joux et la région d'Yverdon. Ce bassin de recrutement est en fait identique à celui identifié lors de l'enquête auprès des hypermarchés français ; en effet, au cours de leur déplacement, les consommateurs suisses combinent différents types de consommation dans la même journée afin de rentabiliser leur voyage. 


\section{Conclusion}

24 espaces. Les mouvements identifiés autour de la frontière confèrent aux espaces transfrontaliers, tantôt un rôle de bassin de vie, tantôt un rôle de bassin d'emploi fondés sur d'intenses relations dont l'existence se matérialise par de multiples migrations de proximité (les temps de transport sont compris entre 30 et $45 \mathrm{mn}$ compte tenu des aménagements et de la situation topographique locale). On prend aujourd'hui la mesure de l'intensité de ces dynamiques transfrontalières locales (frontaliers, consommateurs suisses en France), des synergies économiques (spécialisations industrielles communes ou complémentaires), des perspectives de développement communes et on observe que l'espace transfrontalier franco-suisse de l'arc jurassien fonctionne sur la base d'une succession de bassins de vie qui se traduisent par une structuration tout à fait originale révélant de manière informelle des espaces transfrontaliers dissymétriques, mais très fortement structurés.

Les pôles de Morteau et de Pontarlier sont ceux qui profitent le plus des dynamiques décrites, à la fois pour des raisons de taille conditionnant la présence de services, d'accessibilité mais aussi en raison de la présence de bassins de population suisses importants et dans une moindre mesure de proximité socio-culturelle.

L'existence de ces bassins transfrontaliers nous amène à poser un certain nombre de constats au regard des nombreux problèmes non résolus qui apparaissent de part et d'autre de la frontière :

- la gestion des ressources humaines est faiblement coordonnée : la main-d'œuvre française ne bénéfice d'aucune sécurité d'emploi, les actions de formation ne sont pas forcément complémentaires, la reconnaissance des diplômes n'est pas toujours effective sauf dans le cadre de projets de coopération particuliers ${ }^{7}$;

- l'absence de transports collectifs transfrontaliers demeure un frein à l'organisation rationnelle des flux ;

- la définition d'un bassin d'emploi transfrontalier n'est pas encore admise ;

- l'accès aux services médicaux fait l'objet d'une reconnaissance de la part de la sécurité sociale en France, mais au coup par coup ${ }^{8}$, et sur la base des tarifs français...,

- la faible prise en compte des services présents de part et d'autre de la frontière peut entraîner des doublons et des surcoûts ${ }^{9}$.

L'inévitable intégration de la Suisse à l'Europe apportera-t-elle des solutions, les flux mentionnés se renforceront-ils ou au contraire seront-ils atténués par la disparition de la frontière, autant de question que ne cessent de se poser les aménagistes français et suisses en charge de la gestion de ces territoires. Ces incertitudes expliquent certainement la prudence avec laquelle chacun se penche sur les problèmes de coopération et sur la définition d'aires transfrontalières cohérentes. 


\section{BIBLIOGRAPHIE}

[1] Chevailler J.-C., Sordoillet G. (1990). - « Les travailleurs frontaliers franc-comtois en Suisse », rapport GIMM-CETAP, Université de Franche-Comté, Besançon.

[2] Chevailler J.-C., Sordoillet G., Quillery P. (1991). - « Frontaliers et frontières », in Images de Franche-Comté $\mathrm{n}^{\circ}$ 3, Université de Franche-Comté, Besançon.

[3] Chevailler J.-C., Sordoillet G. (1991). - « Frontaliers : quelque chose à déclarer », in Reflets de l'économie franc-comtoise $n^{\circ} 46$, INSEE, Besançon.

[4] Chevailler J.-C., Gillon P. (1995). - « Les dynamiques économiques et spatiales dans l'Arc jurassien ", in La Suisse et la coopération transfrontalière : repli ou redéploiement ?, sous la direction de Leresche J.-P. et Levy R.

[5] Mauger M. (1995). - « La Suisse, terre d'accueil et partenaire des Francs-comtois », in La Tribune du 26/10/95.

[6] Daviot J.-M., Gillon P. (1995). - « Les liens commerciaux avec la Suisse », in Images de FrancheComté $\mathrm{n}^{\circ} 12$, Université de Franche-Comté, Besançon.

[7] Lechot G., Lecoq B., Pfister M. (1995). - « Horlogerie franco-suisse : des trajectoires divergentes ", in Quel développement pour l'Arc jurassien ?, IRER, Neuchâtel.

\section{NOTES}

1. Les bourses aux affaires transfrontalières régulièrement organisées deux fois par an, tantôt à Vallorbe, tantôt à Pontarlier montrent qu'environ $25 \%$ des annonces présentées (environ 200) à chaque session, relèvent du secteur de la sous-traitance.

2. D'après une enquête de la Coop sur le tourisme d'achat, in « Coop informe », 1996.

3. Télévision Suisse Romande, émission « À bon entendeur ", 23/09/97.

4. Enquête réalisée par D. VERDIER dans le cadre d'un mémoire de Maîtrise en géographie.

5. Nous nous appuyons côté français sur les données d'Inventaire Communal, et côté suisse, de manière plus empirique, sur les résultats de l'enquête déjà citée.

6. Enquête Minitel, réalisée en février 1997.

7. École d'Ingénieur de Saint-Imier/IPSé de Sévenans : échange d'enseignants et reconnaissance d'un titre universitaire eurocompatible pour l'école de Saint-Imier.

8. C'est le cas entre la commune de Bois-d'Amont et l'hôpital de la Vallée depuis 1965.

9. Le projet de piscine à Morteau paraît déplacé lorsqu'on considère la présence de piscines à La chaux-de-Fonds. 


\section{RÉSUMÉS}

Les frontières nationales symbolisent les ruptures entre des systèmes économiques différents. Les conséquences sont nombreuses, elles affectent les espaces limitrophes en déclenchant de nombreux flux qui à leur tour influencent la structuration de ces espaces. Le massif du Jura franco-suisse est à ce titre exemplaire, et nous avons essayé de saisir l'importance de ces flux, au travers de quelques exemples, qui montrent l'existence de bassins de vie et de bassins d'emploi transfrontaliers dans le cadre desquels se déroulent ces migrations.

National borders symbolize the ruptures between different economic systems. The consequences are numerous, they affect adjacent spaces by initiating many flows which in turn, influence the structure of these spaces. The Franco-Swiss Jura massif is a prime example, and we have tried to grasp the importance of these flows, through some examples, which show the existence of concentrations of society and pools of cross-border employment which these migrations exist.

Die nationalen Grenzen symbolisieren die Brüche zwischen verschieden-artigen Wirtschaftssystemen. Die Folgen sind zahlreich; sie beeinflussen die Grenzraüme, indem sie zahlreiche Ströme auslösen, die ihrerseits die Strukturierung dieser Räume beeinflussen. Das französisch-schweizerische Juramassiv ist dafür beispielhaft und es wird hier versucht, die Bedeutung dieser Ströme zu erfassen - mit einigen Beispielen, welche die Existenz von grenzüberschreitenden Wohn - und Arbeitsbereichen zeigen, in deren Rahmen sich die Wanderungen abspielen.

\section{INDEX}

Keywords : border, cross-border employment area, cross-border residential area, daily migrations, flow, Franco-Swiss Jura massif

Schlüsselwörter : Französisch-schweizerisches Juramassiv, Grenze, Grenzüberschreitende Wohn - und Arbeitsbereiche, tägliche Pendelwanderung, Wanderungs-Strom

Mots-clés : bassins de vie, bassins d'emploi, flux, frontière, Massif du Jura franco-suisse, migrations journalières

\section{AUTEUR}

\section{ALEXANDRE MOINE}

IRADES@THÉMA UPRESA 6049 - Université de Franche-Comté - 32, rue Mégevand 25030 Besançon Cedex 